\title{
Erratum
}

\section{Erratum. Adiponectin Prevents Diabetic Premature Senescence of Endothelial Progenitor Cells and Promotes Endothelial Repair by Suppressing the p38 MAP Kinase/p16 ${ }^{\text {INK4A }}$ Signaling Pathway. Diabetes 2010;59:2949-2959 \\ DOI: 10.2337/db16-er10b}

In the article listed above, the quantitative bar graph in Fig. $5 C$ was from real-time PCR analysis using SYBR Green dye, whereas the gel images inserted in this panel were from a separate set of reverse transcription (RT)-PCR analyses to confirm the specificity of the primers used, but not for real-time PCR. An additional lane at the right end of the gels (lane 7) was erroneously included. The revised version of Fig. $5 \mathrm{C}$ includes corrected gel images. The dividing line between lanes 5 and 6 of p16 PCR products shows splicing within the same gel. In the legend of Fig. $5 C$, the statement "The representative gel is shown on the top of the bar chart" is now changed to "The gel images on the top of the bar chart are RT-PCR products for p16 and actin." The authors apologize to the readers for any confusion.
Junlei Chang, Yiming Li, Yu Huang, Karen S.L. Lam, Ruby L.C. Hoo, Wing Tak Wong, Kenneth K.Y. Cheng, Yiqun Wang, Paul M. Vanhoutte, and Aimin Xu

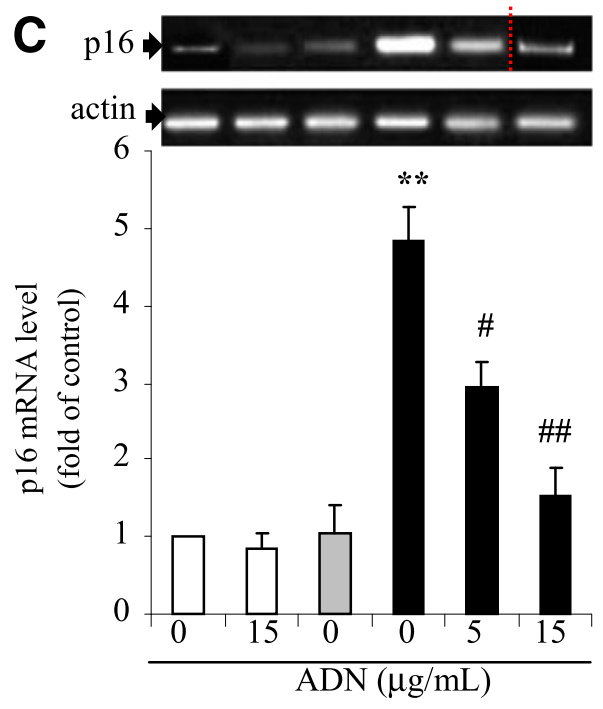

